\title{
Le Schopenhauer de Huysmans et Céard:deux avatars littéraires de la résignation
}

\section{Roderick Cooke}

\section{(2) OpenEdition}

1 Journals

\section{Édition électronique}

URL : http://journals.openedition.org/studifrancesi/16095

DOI : 10.4000/studifrancesi. 16095

ISSN : 2421-5856

Éditeur

Rosenberg \& Sellier

\section{Édition imprimée}

Date de publication : 1 juillet 2019

Pagination : 56-68

ISSN : 0039-2944

\section{Référence électronique}

Roderick Cooke, "Le Schopenhauer de Huysmans et Céard:deux avatars littéraires de la résignation », Studi Francesi [En ligne], 187 (LXIII | I) | 2019, mis en ligne le 01 avril 2020, consulté le 24 janvier 2021. URL : http://journals.openedition.org/studifrancesi/16095 ; DOI : https://doi.org/10.4000/ studifrancesi. 16095

\section{(c) (i) (9)}

Studi Francesi è distribuita con Licenza Creative Commons Attribuzione - Non commerciale - Non opere derivate 4.0 Internazionale. 


\title{
Le Schopenhauer de Huysmans et Céard: deux avatars littéraires de la résignation
}

\begin{abstract}
Examining both explicit and implicit references to Schopenhauer's philosophy in J.-K. Huysmans' À rebours (1884) and Henry Céard's Une belle journée (1881), I argue that the two authors both created a version of it, in the midst of the 'Schopenhauérisme' craze in 1880s France, that validated their shared belief in resignation as an ethical category, and demonstrated the importance of resignation through the psychology of the two novels' protagonists. In so doing, they modified the philosopher's ideas both in line with their own existing beliefs, and in such a way as to allow the narrativization of philosophy in the naturalist novel. Whereas the intellectually limited protagonist of Une belle journée, Ernestine Duhamain, finds minor solace in a philosophical conclusion taken from Schopenhauer (I demonstrate the novel's connection to the philosopher through a previously-undiscussed intertext with Céard's article on Schopenhauer published the same year), Des Esseintes' more overt engagement with Schopenhauer in À rebours brings instead suffering, announcing Huysmans' future conversion to Catholicism. In their different ways, the two novels illustrate Céard's and Huysmans' attempts to differentiate their writing from that of Zola, who was harshly critical of Schopenhauer in his own La Joie de vivre. They also raise wider questions about the relationship between philosophy and literature, specifically the possible disjunction between the general abstraction of the former and the defined characters, plots and environments of the latter.
\end{abstract}

On lit beaucoup plus À rebours (1884) de J.-K. Huysmans qu'Une belle journée (1881) d'Henry Céard, et pourtant ces deux romans des «petits naturalistes», comme les nommait le critique implacable Ferdinand Brunetière, ont bien des points en commun. Cela vaut non seulement sur le plan sociologique, au niveau de la maigre renommée des deux auteurs dans l'orbite et l'ombre zoliennes à l'époque de leur publication, mais aussi sur celui de leur intarissable amitié qui durera jusqu'à la mort de Huysmans en 1907. Tout autant que ces faits annexes, les aspects thématiques et formels d'À rebours et d'Une belle journée se rapprochent, par exemple dans la restriction du champ romanesque à un nombre minime de personnages significatifs - un pour Huysmans, deux pour Céard - et d'espaces, puisque presque l'intégralité d'À rebours se déroule dans la maison de Des Esseintes à Fontenay-aux-Roses, alors que Céard enferme Ernestine Duhamain et Trudon dans un cabinet particulier de restaurant à Bercy pendant la moitié d'Une belle journée.

Le rapprochement que je ferai ici entre les deux œuvres appartient plutôt au domaine de la philosophie, à savoir celle d'Arthur Schopenhauer, philosophe qui intéressa Huysmans et Céard comme bon nombre de leurs contemporains en France. L'intérêt porté à Schopenhauer outre-Rhin date des années 1850, et au moment de la sortie des deux romans qui me préoccupent, les traductions et même les parodies prolifèrent en France. Citons notamment La Philosopbie de Schopenhauer (1874) de Théodule Ribot, et les Pensées, maximes et fragments (1880) de Jean Bourdeau. C'est ce dernier florilège que le groupe de Médan semble avoir pratiqué le plus: ainsi, Céard écrira à Zola en janvier 1880 qu'il en a obtenu un exemplaire et le lui prêtera 
lors de leur prochaine retrouvaille parisienne $e^{1}$. Il faut souligner que la traduction du magnum opus de l'Allemand, Le Monde comme volonté et comme représentation, ne sera accomplie qu'en 1886; la publication eut lieu à Bucarest sous les auspices de Jean-Alexandre Cantacuzène. Cela implique que le Schopenhauer des romanciers naturalistes est inéluctablement partiel, la majeure partie de sa pensée étant encore inaccessible aux seuls francophones. Schopenhauer tel qu'on le trouve dans $\grave{A} r e-$ bours et dans Une belle journée est autant une création ou reconstitution des auteurs respectifs qu'il n'est un penseur ou un système autonome (mais pourrait-il en être autrement, quel qu'aurait été l'état des traductions contemporaines?).

Je suggérerai dans l'analyse qui suit que, malgré d'importantes différences au niveau de la narrativisation de Schopenhauer et des interprétations personnelles de sa philosophie par les deux auteurs, ce qui unit Une belle journée et À rebours est leur manière de déployer le philosophe comme suppôt de la résignation telle que les protagonistes l'incarnent. C'est-à-dire que, pour Jean Floressas des Esseintes tout comme pour Ernestine Duhamain, un Schopenhauérisme (c'est le terme des années 1880 qui revient) plus ou moins explicite est à la base de leurs réflexions sur la vie, son manque de satisfactions, et surtout l'abnégation par laquelle on devrait y répondre.

Ces parallèles philosophiques seront sondés par des commentaires textuels, se concentrant sur les passages des deux romans dans lesquels l'allusion à Schopenhauer est le plus clairement affichée par Céard et Huysmans. Celui-ci fera figurer le nom même, à côté d'une version des idées, de l'Allemand à deux reprises dans le texte d'À rebours, et la critique n'a pas manqué d'examiner ces pages. Mais chez Céard, malgré l'évident intérêt qu'il porte aux idées schopenhaueriennes (nous avons déjà constaté sa propriété du livre de Bourdeau), le nom du philosophe ne figure jamais, ni dans Une belle journée, ni dans ses autres écrits imaginatifs. Cela a porté les auteurs des assez rares travaux consacrés au roman à écarter l'hypothèse Schopenhauer'. RenéPierre Colin affirme que «l'interprétation de Céard [...] laisse peu de traces dans son œuvre puisque ses héros accablés par l'ennui, les misères de la vie quotidienne, la bêtise, n'ont guère la force d'aménager leur existence pour y réduire la douleur. Ils ne vivent en fait jamais le moment présent, mais s'emploient simplement à découvrir leur salut dans de pauvres accommodements» ${ }^{2}$. Colin envisage donc les éventuelles 'traces' de Schopenhauer dans la fiction de Céard à travers une optique de la fidélité, où les personnages assumeraient des comportements éthiques conformes à ceux préconisés dans le Livre IV du Monde comme volonté et comme représentation.

Il est certes vrai, non seulement que les traces explicites de l'Allemand dans Une belle journée sont absentes mais aussi, comme le souligne Colin, qu'une éthique authentiquement schopenhauerienne l'est tout autant. Pourtant, en abordant la question par la prémisse de la création d'un Schopenhauer céardien qui, lui, serait inséré dans le roman, l'examen peut se poursuivre de façon plus fertile. Il s'agira donc d'abord de clarifier en quoi pourrait consister le Schopenhauer de Céard, si ce n'est pas un facsimilé du 'vrai', avant de partir à sa recherche dans le texte d'Une belle journée.

Bien que Céard n'ait jamais nommé Schopenhauer dans ses romans, il en est tout autrement de son journalisme. Et Céard, quant à sa productivité, était bien plus journaliste que romancier. Alors qu'on ne peut lui attribuer que deux romans - Une

(1) Cité dans T. WEst, Schopenhauer, Huysmans and French Naturalism, «Journal of European Studies» 1, 4, 1971, pp. 313-324, p. 316. Lettre publiée dans Lettres inédites de Céard à Zola, éd. C. Burns, Paris, Nizet, 1958, lettre 44.

(2) R.-P. Colin, Schopenhauer en France: Un mythe naturaliste?, Lyon, Presses Universitaires, 1979, p. 160. 
belle journée sera finalement suivi, vingt-cinq ans plus tard, de Terrains à vendre au bord de la mer -, ses articles dans la presse parisienne, (et, plus rarement, londonienne et argentine, inter alia) s'énumèrent plutôt par centaines. Au sein de ce corpus journalistique, la série d'articles la plus pertinente est celle des Portraits littéraires. Malgré leur titre, la seule constante 'littéraire' qu'on y remarque, c'est la technique de Céard en décrivant les personnalités de son choix, puisque parmi celles-ci se trouvent aussi bien des musiciens tels que Wagner que des romanciers tels que Laclos (auteur fétiche de Céard)... et des philosophes tels que Schopenhauer.

D'ailleurs le jeune naturaliste a publié son portrait du philosophe deux fois, d'abord dans «L'Express» du 8 août 1881, puis (en version légèrement remaniée et intitulée Clowns et philosophie) dans «Le Siècle» du 19 octobre 1888. Le fait que Céard a fait paraître une étude sur Schopenhauer la même année que la parution d'Une belle journée est en lui-même frappant, mais les détails du texte le sont bien plus. Commençons par citer ce passage:

Il exhorte à se tenir dans son milieu, et, puisqu'il est en dehors de nos facultés d'échapper aux lois dominatrices et inconnues qui régissent nos volontés et déterminent nos actes, il enseigne d'entrer tous les jours plus profondément dans la connaissance de son néant, de façon à ne jamais être dupe au moins de ses propres entraînements et de ses naturelles erreurs. Puisque le mal s'impose, il s'agit d'en profiter et d'en extraire la plus grande somme de bonheur possible. Tirer parti de tout, en vue de la diminution des souffrances, c'est le dernier mot de sa philosophie, la conclusion de toute son œuvre $[\ldots]^{3}$ (je souligne).

J'ai souligné certaines phrases et tournures ci-dessus pour des raisons qui deviendront claires une fois que le texte d'Une belle journée sera abordé. Mais même en se restreignant au seul article, ce qui en ressort, c'est la lecture de Schopenhauer comme apôtre de la résignation. Alors que Le Monde comme volonté et comme représentation comprend quatre Livres, respectivement consacrés à l'épistémologie, l'ontologie, l'esthétique et l'éthique, ce n'est que le dernier de ces domaines qui intéresse réellement Céard. L'esthétique, chez cet esthète polymathe, de l'Allemand n'est pas effleurée, l'épistémologie guère plus (malgré 'connaissance de notre néant'), et les allusions ontologiques - 'lois dominatrices et inconnues', 'le mal s'impose' - sont surtout mises au service de la conclusion éthique qui en découle.

Bien sûr, comme nous l'avons constaté, Céard n'a pas lu l'intégrale de Schopenhauer. Peut-on mettre sur le seul compte de Jean Bourdeau et ses Pensées, maximes et fragments la version de Schopenhauer que Céard revendique dans ce 'Portrait littéraire'? Pas entièrement, mais en parcourant ce volume et en notant les choix du traducteur, il est évident qu'ils ont modulé les pensées du romancier. Ainsi la douleur et la désillusion viennent en tête du florilège, et la section la plus longue est consacrée à la «Métaphysique de l'amour» de Schopenhauer. Marc Smeets le signale, «l'anthologie de Bourdeau [...] donne une image réductrice de la pensée de Schopenhauer» ${ }^{4}$. La version qu'en donne Bourdeau ressemble plus aux moralistes de la tradition française qu'au bâtisseur de systèmes de son homologue allemande. La section s'ouvre sur une épigraphe de Chamfort, ce qui a dû pousser Céard à affirmer dans son article que «Schopenhauer a la phrase nette, courte, acérée $[. .$.$] cela possède la vivacité et$ le mordant de Chamfort».

(3) Cité dans C. Burns, Henry Céard et le naturalisme, Birmingham, John Goodman and Sons, 1982, pp. $172-173$.

(4) M. SmeETs, Huysmans, Maupassant et Schopenhauer: Note sur la métaphysique de l'amour, in Guy de Maupassant, éd. Noëlle Benhamou, New York et Amsterdam, Rodopi, 2007, p. 22. 
Par contre, ce qu'on ne trouve pas chez Bourdeau et qui est une dominante dans la reprise céardienne de Schopenhauer, c’est la métaphore médicale filée. Sous la plume du romancier naturaliste, le philosophe devient «un médecin, un expérimentateur, un physiologiste». Pour Céard, il s'agit d'un penseur qui cherche «d'une façon certaine les moyens de rendre l'homme moins douloureux et la vie plus supportable. Il ne se croit pas en droit d'affirmer l'existence d'une justice supérieure, et, s'en rapportant consciencieusement aux constations de la science, ne préjuge de l'existence ou de la non existence d'aucune divinité». Autrement dit, c'est un positiviste à ranger aux côtés de Littré, Berthelot, ou surtout de... Claude Bernard.

Et voilà bien l'ironie suprême du portrait de Schopenhauer qu'esquisse Céard. Nous sommes en 1881, l'année après la parution en volume du Roman expérimental de Zola . Quatre mois avant la publication de ce Portrait littéraire, Zola donnait au «Figaro» son article Céard et Huysmans, où il faisait un compte rendu positif des récemment parus En ménage de celui-ci et Une belle journée de celui-là, ainsi qu'une réflexion plus large sur le soi-disant 'groupe de Médan', dont la notoriété était à son apogée - 1880 n'est pas seulement la date de parution du Roman expérimental, mais aussi des Soirées de Médan, où les trois auteurs figureront aux côtés de Guy de Maupassant, Léon Hennique et Paul Alexis. Dans Céard et Huysmans, Zola prédit qu'Une belle journée sera «une œuvre qui marquera [...] car cette œuvre est d'une philosophie nette et d'un accent personnel» ${ }^{6}$.

Tout cela semble former une constellation d'arguments esthétiques, épistémologiques et sociologiques qui rendent presque banale la métaphore médicale employée par Céard pour caractériser Schopenhauer: ne suit-il pas l'exemple de Zola, son maître à penser, qui avait fait de son essai Le Roman expérimental une paraphrase et commentaire de l'Introduction à la médecine expérimentale de Claude Bernard? En vérité, il n'en est rien. Car, tout comme Brunetière qui est hostile aux deux hommes, Céard, le prétendu disciple 'petit naturaliste' de Zola, a de graves réserves envers la thèse de celui-ci dans Le Roman expérimental. Il s'agit de l'argument décisif contre les prétentions à l'exactitude scientifique du naturaliste aîné, selon lesquelles faire passer un personnage à travers les étapes d'un récit encadré par un milieu soigneusement observé équivaudrait à une expérience en laboratoire. Céard, encore fidèle à l'image publique du maître, gardera ces réserves pour une lettre du 28 octobre 1879 dans laquelle il explique que:

Décidément, il y a un sophisme capital dans votre étude sur le roman expérimental. Claude Bernard, quand il institue son expérience, sait parfaitement dans quelles conditions elle se produira et sous l'influence exacte de quelles lois déterminées [...] En est-il de même pour le romancier? [...] Les lois du cerveau n'étant que bien vaguement formulées, au lieu d'aboutir à une réalité scientifique, comme Claude Bernard, il aboutit simplement à une hypothèse, vraisemblable sans doute, mais qu'il ne peut appuyer sur aucun fait et qui laisse prise à toutes les discussions ${ }^{7}$.

Mais voilà que, presque deux ans plus tard et alors que cet argument aura été entériné par les adversaires littéraires et intellectuels du naturalisme, Céard offre une

(5) L'essai éponyme avait d'abord paru en été 1879, aussi bien dans «Le Messager de l'Europe» de SaintPétersbourg que dans «Le Voltaire» à Paris.

(6) Cet article sera repris dans le volume Une campagne plus tard en 1881; c'est la version que je cite ici.

E. Zola, Céard et Huysmans, in Une campagne, Paris, Fasquelle, 1881, pp. 251-261, p. 260.

(7) Cité dans H. Mitterand, Notice, in E. Zola, Euvres complètes, vol. 10, éd. H. Mitterand, Paris, Cercle du Livre Précieux, 1969, pp. 1403-1404. 
vision de Schopenhauer dans laquelle il ressemble étonnamment à ce même Claude Bernard, après avoir commenté à Zola dans une autre lettre que «[1]e système littéraire actuel a des points communs avec la doctrine de ce philosophe». Et Céard est suffisamment convaincu de ce portrait pour le rééditer en 1888. Comment expliquer cet apparent vacillement?

Schopenhauer a peut-être des affinités stylistiques avec Chamfort au sens de Céard, mais il s'agit bien d'un philosophe et non d'un littérateur. C'est-à-dire que, alors que les revendications de Zola quant au statut intellectuel du roman ne convainquent pas son jeune collègue, le statut intellectuel de la philosophie ne pose pas le même problème. Il dira donc dans le Portrait littéraire de Schopenhauer qu'il «sait les misères des fonctions, l'infériorité des organes, les faiblesses du cerveau, et ne leur demande par conséquent rien au-delà de leur capacité et de leurs forces». Ce même cerveau dont Céard mettait en cause la connaissance pour critiquer la thèse expérimentale de Zola, il en attribue le savoir à Schopenhauer sans hésitation, mettant cet organe sur le même pied que les autres par le zeugme qui dépend du verbe 'sait'. C'est précisément cette parité entre cerveau et autres organes qu'il avait niée à Zola en 1879.

$\mathrm{Si}$ «le système littéraire actuel a des points communs avec la doctrine de ce philosophe», ce n'est pas, semble-t-il, dans le roman zolien qu'ils existaient pour Céard. Je veux suggérer qu'il songeait plutôt au sien, sur le point d'être publié alors qu'il écrivait cette phrase. Dans Une belle journée et surtout dans sa conclusion, une lecture fouillée permet de déceler des traces du Schopenhauer céardien tel que l'avons rencontré dans son Portrait littéraire.

Résumons d'abord le récit de ce roman dont Zola a dit «qu'il sera difficile désormais de dépasser Céard, d'écrire tout un volume avec un fait plus simple [...] il s'est demandé bonnement si, de l'aventure la plus banale, il ne serait pas possible de tirer de l'intérêt, de dégager une émotion profondément humaine»". L'aventure banale est celle de Mme Duhamain, bourgeoise frustrée du quartier de Picpus qui donne rendez-vous à son voisin du dessus, un célibataire nommé Trudon, comptant passer la belle journée ironiquement éponyme avec lui suite à une soirée de sortie humiliante avec son mari. Mais, pendant plus de 150 pages, ils se retrouvent coincés par la pluie dans le cabinet particulier du restaurant des «Marronniers» à Bercy, le désir initial cédant rapidement la place à la gaucherie et au désenchantement. Rien ne se passera entre eux, et au terme d'un voyage en fiacre Ernestine rentre chez elle et retrouve M. Duhamain, alors que Trudon rentrera plus tard avec une des femmes dont il a plus l'habitude. Maxime Gaucher, dans sa 'Causerie littéraire' pour la «Revue bleue», a repris des aspects du jugement de Zola, mais sur un ton âprement satirique:

Et le grand Zola dit au jeune Céard: Tu es le bien-aimé dans la foule de mes disciples [...] car aucun des autres n'a montré une soumission si complète [...] Une belle journée réalise ma formule dans sa simplicité et son intégrité. Ni poésie, ni sentiment, ni intérêt, rien, rien, absolument rien! Bravo! [...] - Ainsi a parlé le grand Zola et il a dit vrai. Non, il n'y a rien dans ce volume qu'une série de petits documents insignifiants, de petites observations banales, parfois vulgaires et pis que vulgaires ${ }^{10}$.

L'opinion de Gaucher incarne la réaction critique contemporaine dont les séquelles ont longtemps maintenu Une belle journée dans l'oubli. À partir des années

(8) Lettre à Zola du 13 janvier 1880, citée dans CoLIn, Schopenhauer en France cit., p. 157.

(9) E. Zola, Une campagne cit., pp. 258-259.

(10) M. GAuCHER, Causerie Littéraire IV, «La Revue politique et littéraire: revue des cours littéraires» 19 , éd. E. Yung, $3^{\text {eme }}$ série, $1^{\text {ere }}$ année, 7 mai 1881, p. 604. 
1950, dans les universités anglophones, des chercheurs tels que Ronald Frazee, Murray Sachs et surtout C.A. Burns ont commencé à faire sortir l'œuvre de Céard de l'ombre; Burns la réédita chez Slatkine en 1970. Chez les critiques francophones, on peut citer d'abord Pierre Cogny, plus tard René-Pierre Colin, Éléonore Roy-Reverzy et, plus récemment, Carine Goutaland parmi ceux qui ont donné des analyses du roman. La revalorisation critique d'Une belle journée est typiquement passée par l'accent mis sur son proto-modernisme (Goutaland suggère que le roman «annon[ce] l'absurde beckettien et le Nouveau Roman» ${ }^{11}$ ), ou bien sur sa réalisation du rêve flaubertien du 'livre sur rien'. William W. Thomas fond ces deux camps en un: d'une part, il soutient que «the modern reader will recognize in Une belle journée many characteristics of the contemporary novel, particularly the nouveau roman [...] the technique of portraying the characters through a fairly elaborate process of stimulusthought-response calls to mind especially the process of conversation and sous-conversation used by Nathalie Sarraute» ${ }^{12}$, mais de l'autre son étude trace les intertextes et les réductions opérées par Céard par rapport à Madame Bovary.

Que ce soit Zola, premier critique à prendre Une belle journée au sérieux, ou les universitaires des dernières décennies qui ont pris sa relève, on s'accorde donc pour faire de Céard un psychologue plus assidu et, peut-être, plus nuancé que Zola lui-même. Mais comment cela peut-il étayer une lecture schopenhauerienne du roman? Pourquoi Céard aurait-il rapproché «le système littéraire actuel» et la pensée de l'Allemand?

Ces questions trouvent leur réponse dans un passage de la conclusion du roman qui est souvent cité, mais dont je compte donner une interprétation nouvelle:

Pendant qu'elle coiffait son filet de nuit où ses cheveux tout blonds tombaient retenus par les larges mailles blanches, des philosophies s'éveillèrent dont elle eut obscurément conscience. Elle comprit que la misère des cœurs résulte non pas de la douleur continue qui les poigne, mais de l'effort qu'ils font pour échapper à leur condition. [...] Elle devina quelle ampleur de sottise se manifeste dans les continuelles révoltes contre cette loi de la médiocrité universelle qui pareille à la gravitation et despotique autant que la pesanteur, ploie le monde et le soumet à son ordonnance: cette nécessité lui apparut qu'il fallait se tenir à sa place et tâcher de s'y faire tout petit pour diminuer les risques d'aventures et provoquer le moins possible les déconcertants déclanchements de la fatalité $[. .$.$] le mieux consistait à s'étendre dans une platitude définitive.$ Autant valait rechercher par inclination un état où elle serait inévitablement ramenée par force. C'était le calme assuré, d'abord, et peut-être que de la continuité même naitrait à la fin une jouissance ${ }^{13}$. (je souligne)

Dans le Portrait littéraire de Schopenhauer qui parait trois mois après Une belle journée, Céard dit du philosophe qu'«[i]l est bien convaincu que les êtres humains, par le fait même de leur existence, sont soumis à une loi de misère écrasante, despotique comme la gravitation, inévitable comme la pesanteur». La transposition de la phrase est nette, et confirme la présence déguisée d'un Schopenhauer céardien à la fin d'Une belle journée. La Volonté schopenhauerienne, qui dans Le Monde comme volonté et comme représentation est à la base du système ontologique - pour Scho-

(11) C. Goutaland, Dire la bricole: tours et détours linguistiques dans "Une belle journée” d'Henry Céard, in Des mondes bricolés? Arts et sciences à l'épreuve de la notion de bricolage, sous la direction de F. Odin et C. Thuderoz, Lausanne et Lyon, Presses Polytechniques et Universitaires Romandes, 2010, pp. 295-307, p. 304.

(12) W.W. Thomas, Henry Céard's “Une belle journée”, «Nineteenth-Century French Studies» 5, 1977, pp. 328-340, p. 339 .

(13) H. CÉARD, Une belle journée, Paris, Charpentier, 1881, pp. 338-340. 
penhauer, il n'existe rien d'autre de fondamental que la Volonté, dont tout être et objet n'est qu'une représentation dans le temps et l'espace - Céard la restreint assez logiquement au champ humain, et s'en sert pour expliquer les sottises des comportements individuels. D'où la futilité de la 'révolte'. Dans le roman, il s'agit d'un «idéal [...] meurtrier» à éviter, alors que dans la chronique l'accent est plus épistémologique puisqu'il s'agit «d'entrer tous les jours plus profondément dans la connaissance de son néant, de façon à ne jamais être dupe au moins de ses propres entraînements et de ses naturelles erreurs». Cela rejoint l'impératif de «se tenir à sa place et tâcher de s'y faire tout petit pour diminuer les risques d'aventures» qui se suggère à Mme Duhamain.

Il faut donc contester l'affirmation de Colin, citée ci-dessus, selon laquelle «l'interprétation de Céard [...] laisse peu de traces dans son œuvre», aussi bien que celle de Ronald Frazee qui évoque l'hypothèse de Schopenhauer pour l'écarter immédiatement: «le roman [...] offrait l'occasion d'étudier avec minutie une situation toute banale; il permettait d'atteindre, à partir de cette situation et des mouvements de pensée qu'elle faisait naître, une conclusion logique. Et bien qu'on puisse voir là Céard interprétant Schopenhauer à sa façon, le roman est plutôt triste que pessimiste» ${ }^{14}$. Dans les deux cas, c'est l'absence d'un pessimisme existentiel sans bornes, où d'une abnégation sincère du vouloir-vivre, dans Une belle journée qui dépiste le critique, mais nous avons vu que l'optique de la fidélité au Monde comme volonté et comme représentation n'est pas celle qui rend le mieux compte du travail de Céard. C'est justement sa façon de construire un autre Schopenhauer, apte à mimer ses propres intuitions philosophiques ainsi qu'à fournir des philosophèmes prêts à insérer dans le texte romanesque, qui en fait l'originalité.

Dans la lecture que fait Céard de la pensée schopenhauerienne, entre fiction et journalisme, les liens sont donc étroits et révélateurs; cependant il faut également souligner les modifications subtiles mais significatives que la narrativisation fait subir au Schopenhauer céardien. Par exemple, on constate que, même dans l'intertexte central qui permet de relier le Portrait littéraire et les dernières pensées d'Ernestine Duhamain, la loi quasi-gravitationnelle de Schopenhauer est, dans celles-ci, une «loi de la médiocrité universelle», alors que dans celui-là c'est «une loi de misère écrasante». Préférant la médiocrité à la misère, le roman offre un aperçu moins désolant, plus aplati de la métaphysique schopenhauerienne telle que Céard la concevait: comme l'a fait remarquer Rudy Steinmetz à propos de ce même philosophe et $\grave{A}$ rebours, «[1]a philosophie n'est pas simplement devant la littérature comme un réservoir où celle-ci viendrait puiser ses ressources thématiques. Elle fait l'objet d'un travail modificateur de la part du discours où elle est insérée» ${ }^{15}$. C'est sûrement le cas parce que la différence conceptuelle entre roman et chronique est bien celle de la spécificité.

Par cela j'entends que dans un compte rendu abstrait de la philosophie - n'importe laquelle - tel que nous en voyons dans le Portrait littéraire, l'ordre général des idées peut être respecté, les déclarations universelles. Mais, dès que celles-ci deviennent philosophèmes dans un roman, elles deviennent liées à des personnages, des milieux et à un récit définis, et leur étendue est presque obligatoirement bornée par le processus narratif. La vie bourgeoise d'Ernestine Duhamain sied mal à la notion de «misère écrasante», alors que bourgeoisie et 'médiocrité' sont quasiment synonymes pour les auteurs réalistes et naturalistes du dix-neuvième siècle. De même, alors que

(14) R. FrazeE, Henry Céard, idéaliste détrompé, Toronto, University of Toronto Press, 1963, pp. 108-109.

(15) R. Steinmetz, Huysmans avec Schopenhauer: le pessimisme d' “Á rebours”, «Romantisme» 61, 1988, pp. 59-66, p. 64. 
l'article de Céard sur Schopenhauer parle de «naturelles erreurs», ce qui pourrait signifier n'importe quelle faute, le roman préfère «les risques d'aventures», évoquant ainsi la faute la plus menaçante pour une femme telle qu'Ernestine, l'adultère, celle qu'elle vient d'éviter de justesse. Les contextes fictif, culturel et historique viennent définir, préciser mais aussi limiter les maximes philosophiques: et, je souligne, tout cela s'ajoute à l'autre écart entre la pensée de Schopenhauer comme 'chose en soi' et la version qu'un Céard en crée par la suite.

Proto-moderniste ou psychologue, Céard a pu l'être pour certains lecteurs, mais il s'ensuit de l'analyse qui précède que l'importance du milieu et son effet sur les personnages dans sa fiction ne devrait pas non plus être négligée. Rappelons que le romancier présente Schopenhauer comme un philosophe qui «exhorte à se tenir dans son milieu», tout comme Mme Duhamain se rend compte que «la misère des cœurs résulte non pas de la douleur continue qui les poigne, mais de l'effort qu'ils font pour échapper à leur condition». Dans les deux cas, le milieu est implicitement assimilé au positionnement social, celui de résidente du quartier Picpus, par exemple, ou encore celui de femme d'architecte respectée. C'est-à-dire que la douleur métaphysique et universelle de Schopenhauer devient individuée dans sa redite céardienne, associée à des personnages et à des réalités socio-économiques définis.

L'éthique subit le même sort: alors que Céard annonce dans l'article que «[t]irer parti de tout, en vue de la diminution des souffrances, c'est le dernier mot de sa philosophie», cette idée est transposée dans le roman de cette façon-ci. Au moment où les deux personnages commandent à déjeuner, Mme Duhamain à qui Trudon devient déjà rébarbatif décide que «[p]uisque, sans qu'elle sût bien pourquoi, elle était montée dans ce cabinet particulier, le mieux était de prendre son parti d'un rendez-vous qu'elle avait sottement accepté. Même elle trouva très comique de profiter de la circonstance pour faire un "bon petit déjeuner" $>^{16}$. C'est en songeant à commander un gigot d'agneau aux frais du séducteur défaillant qu'Ernestine 'tire parti' de sa situation, détail qui introduit un écart ironique entre la généralité du philosophème et la petitesse de sa narrativisation.

David Baguley observe que «[b]oth characters seem to take on to some degree the philosophical attitudes of their author, Mme Duhamain in particular as, at the end of the novel, she meditates upon "cette loi de la médiocrité universelle $[\ldots]$ " ${ }^{17}$, et nous avons vu comment Zola, dès la publication du roman, affirma que «cette œuvre est d'une philosophie nette». La philosophie est effectivement nette, et Baguley associe avec justesse les pensées d'Ernestine avec celles de son créateur, mais sortir des confins du roman confirme que Céard s'est tourné vers Schopenhauer pour les cristalliser. Ainsi le mot de la fin dans le Portrait littéraire - que la doctrine de l'Allemand «s'adresse à l'intelligence dans tout ce qu'elle a de supérieur, flatte l'homme dans tous ses sentiments de liberté» - est effacé par la mise en récit céardienne de son système. Thomas, contrastant le roman avec Madame Bovary, fait remarquer que «Emma possesses the ability to create dreams [...] Mme Duhamain and Trudon almost totally lack this ability», et que ceux-ci sont «two characters who are so dull that the reader takes little personal interest in their lives» ${ }^{18}$. Leurs intelligences n'ont rien de supérieur, et voilà en quoi consiste l'écart entre les deux versions de Schopenhauer que Céard a produites, dans la presse et dans le roman.

(16) H. CÉARD, Une belle journée cit., p. 135.

(17) D. Baguley, A Harmless Liaison: on Henry Céard's "Une belle journée", «Nineteenth-Century French Studies»18, 3/4, 1990, pp. 482-491, p. 488.

(18) W.W. Thomas, Henry Céard's “Une belle journée” cit., pp. 333-334. 
C'est aussi de là, en partie, que l'ironie persistante d'Une belle journée s'alimente: entre l'abstraction philosophique et les pensées d'un Trudon, la possibilité du rire moqueur est inhérente. Doit-on suggérer que ce problème dépasse les pages de ce seul roman? Toute œuvre qui transforme des philosophèmes en narratèmes courtelle le même risque (ou s'offre-t-elle la même opportunité, selon la perspective)? Puisque la philosophie - ou tout au moins une philosophie systématique comme l'est celle de Schopenhauer - opère à l'échelle universelle, de transformer ses préceptes en exemples à l'échelle d'un récit pourrait faire basculer celui-ci dans le domaine ironique sans qu'on le veuille. Qui dit écart dit rapidement ironie.

La narrativisation de Schopenhauer effectuée trois ans plus tard par J.-K. Huysmans nous donne l'occasion de creuser cette question. Et la notion d'intelligence est, ici aussi, au centre du travail. Car voici le passage dans lequel Huysmans déploie Schopenhauer de façon soutenue, en passant d'une méditation sur le catholicisme vers le philosophe:

Ici, Des Esseintes reprenait pied. Certes, il était satisfait de cet aveu de l'ordure sociale, mais alors, il se révoltait contre le vague remède d'une espérance en une autre vie. Schopenhauer était plus exact; sa doctrine et celle de l'église partaient d'un point de vue commun; lui aussi se basait sur l'iniquité et sur la turpitude du monde, lui aussi jetait avec l'Imitation de notre-seigneur, cette clameur douloureuse: «c'est vraiment une misère que de vivre sur la terre!» [...] mais il ne vous prônait aucune panacée, ne vous berçait, pour remédier à d'inévitables maux, par aucun leurre. [...]

Élancée de la même piste que l'Imitation, cette théorie aboutissait, elle aussi, mais sans s'égarer parmi de mystérieux dédales et d'invraisemblables routes, au même endroit, à la résignation, au laisser-faire.

Seulement, si cette résignation tout bonnement issue de la constatation d'un état de choses déplorable et de l'impossibilité d'y rien changer, était accessible aux riches de l'esprit, elle n'était que plus difficilement saisissable aux pauvres dont la bienfaisante religion calmait plus aisément alors les revendications et les colères. Ces réflexions soulageaient Des Esseintes d'un lourd poids; les aphorismes du grand allemand apaisaient le frisson de ses pensées et cependant, les points de contact de ces deux doctrines les aidaient à se rappeler mutuellement à la mémoire, et il ne pouvait oublier, ce catholicisme si poétique, si poignant, dans lequel il avait baigné et dont il avait jadis absorbé l'essence par tous les pores ${ }^{19}$.

Schopenhauer est, dans À rebours, enfermé dans un tête-à-tête avec le catholicisme, et surtout avec L'Imitation de Jésus-Christ (typiquement attribué à Thomas à Kempis autour de 1400). Huysmans établit un dialogisme entre les deux sources, entre la piété médiévale et le pessimisme de l'Allemand, dans l'esprit de son protagoniste. Dans les deux cas, ce qui plait à Des Esseintes, c'est «cette résignation tout bonnement issue de la constatation d'un état de choses déplorable et de l'impossibilité d'y rien changer». L'avantage que le duc accorde au philosophe par rapport à la dévotion d'à Kempis, c'est d'être «la grande consolatrice des intelligences choisies, des âmes élevées», ceux qui n'ont pas besoin de la consolation métaphysique pour apaiser la peine d'un monde de douleur, se targuant de vivre du seul constat pessimiste.

Des Esseintes se range parmi ces «riches de l'esprit», et Huysmans semble avoir été d'accord avec lui. Expliquant la genèse d'À rebours dans la fameuse Préface écrite vingt ans après le roman de 1903, il évoque son récit précédent À Vau-l'eau (1882), dont le protagoniste M. Folantin avait déjà nommé Schopenhauer dans la conclu- 
sion: «Je me figurais un monsieur Folantin, plus lettré, plus raffiné, plus riche et qui a découvert, dans l'artifice, un dérivatif au dégoût que lui inspirent les tracas de la vie et les mœurs américaines de son temps; je le profilais fuyant à tire-d'aile dans le rêve, se réfugiant dans l'illusion d'extravagantes féeries, vivant, seul, loin de son siècle, dans le souvenir évoqué d'époques plus cordiales, de milieux moins vils» ${ }^{20}$. Le contraste entre Des Esseintes et, non seulement Folantin, mais les personnages de Céard dans Une belle journée est donc net. Alors que Folantin, Mme Duhamain et Trudon sont tous trop médiocres pour accéder aux vérités profondes de la doctrine schopenhauerienne telles que Céard et Huysmans les ont entendues, l'aristocrate créé par ce dernier est d'une autre envergure.

La proximité entre auteur et personnage sur la question de Schopenhauer est confirmée par la correspondance de Huysmans. Protestant le portrait à son sens peu flatteur du philosophe que Zola avait donné dans La Joie de vivre en cette même année 1884, son cadet affirme que «tout le côté absolument consolant de cette doctrine et anti-romantique, anti-Wertheriste, bien que vous en puissiez dire, n'y est pas assez, je trouve, exposé. Songez que c'est la théorie de la résignation, la même théorie absolument que celle de l'Imitation de Jésus-Christ, moins la panacée future, remplacée par l'esprit de patience $[\ldots] \gg^{21}$. Comme dans A rebours, le dialogisme et le parallélisme entre Schopenhauer et la sagesse chrétienne sont irréductibles.

Et ce dialogisme se maintiendra dans la pensée de Huysmans jusqu'à sa mort. En 1891, l'année d'avant sa conversion au catholicisme, il déclarait à un destinataire inconnu que «[j]adis, j'ai beaucoup aimé Schopenhauer - aujourd'hui il me désenchante. J'apprécie encore l'exactitude de ses constats, mais le néant de ses conclusions me gêne. Dans l'inintelligible abomination qu'est la vie, il ne peut pas ne rien y avoir» ${ }^{22}$. L'auteur reste entièrement dans le lexique de l'affectivité ('aimé', 'désenchante', 'apprécie', 'gêne', tout cela en deux phrases qui rendent claire la bipolarité de son point de vue) et ne cherche pas à soutenir que Schopenhauer soit inexact. Mais la vérité n'est pas le critère de sélection pour Huysmans: ces vérités sont devenues gênantes, et doivent être repoussées par l'affirmation par laquelle il termine la discussion.

Dans un rapprochement piquant pour notre sujet d'analyse, c'est dans une lettre à Céard un an avant de mourir que Huysmans confirme la persistance de la syzygie Schopenhauer-christianisme, révélant que celle-ci a perduré bien au-delà de la conversion de l'auteur; c'est-à-dire que devenir catholique n'a pas fait oublier Schopenhauer à Huysmans. Quand Céard publie son deuxième et dernier roman, Terrains à vendre au bord de la mer, en 1906 (je l'ai mentionné ci-dessus, le volume se sera fait attendre vingt-cinq ans après la sortie d'Une belle journée), son ami l'en félicite en ces termes: «ce que vous pensez de l'existence et faites dire à Laguépie et montrez dans les funèbres amours de Malbar et de la Trénissan, m'enchante, car je ne puis ne point professer ces mêmes idées, célébrées par Schopenhauer et, avant lui, dans la Bible, par de vieux sages» ${ }^{23}$. Ainsi, quand Huysmans a écrit dans $\grave{A}$ rebours que «les points de contact de ces deux doctrines les aidaient à se rappeler mutuellement à la mémoire», il ne savait pas si bien dire.

Huysmans et Céard se sont fait une image identique de Schopenhauer - un apôtre de la résignation dont les âpres vérités ne sont abordables que par une élite in-

(20) Ibid., p. 58.

(21) ID., Lettres inédites à Emile Zola, éd. Pierre Lambert, Genève, Droz, 1953, p. 99. Lettre de mars 1884.

(22) Cité dans G. Chastel, J.K. Huysmans et ses amis, Paris, Grasset, 1957, p. 283. Lettre de novembre 1891.

(23) Cité dans C. Burns, Henry Céard cit., p. 294. 
tellectuelle - mais seul Huysmans, dans À rebours, a campé un personnage qui puisse en faire partie. Et pourtant, le philosophe réapparaitra à la toute dernière page du roman, et cette réapparition mettra en cause son pouvoir consolateur. Des Esseintes, dont les médecins lui ont affirmé que l'isolement dans la maison de Fontenay est insoutenable et que le retour à la société parisienne s'impose, se résigne à suivre leur conseil, mais celui-ci le plonge dans l'aporie: «Il appelait à l'aide pour se cicatriser, les consolantes maximes de Schopenhauer [...] mais les mots résonnaient, dans son esprit, comme des sons privés de sens; son ennui les désagrégeait [...] il s'apercevait enfin que les raisonnements du pessimisme étaient impuissants à le soulager, que

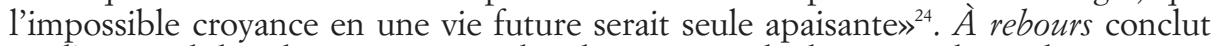
sur l'impossibilité de croire, soit en la religion, soit à la doctrine schopenhauerienne; cependant la préférence antérieure accordée à celle-ci s'estompe, et le catholicisme et son après-vie deviennent la solution, pour l'instant présente uniquement au conditionnel. Cela annonce, bien sûr, l'évolution future de Huysmans dans les domaines esthétique et religieux, vers le 'cycle de Durtal' où le rôle de la religion passera du conditionnel à l'indicatif.

A l'instar d'Une belle journée, À rebours nous montre comment la narrativisation d'un philosophème peut en modifier le sens. Dans le roman de Céard, c'était l'écart entre auteur et personnage qui avait infléchi le sens de l'idée schopenhauerienne vers le particulier et la réalité sociale. Dans celui de Huysmans, le personnage est moins borné, mais Schopenhauer trouve néanmoins un succès moindre au niveau du récit. Selon Colin, «[1]a démarche huysmansienne [...] ne vise à rien d'autre qu'à découvrir un garant philosophique à un pessimisme profond: Huysmans voit dans l'œuvre de Schopenhauer un reflet de sa propre pensée d'autant plus flatteur que le nom du philosophe est familier à une élite» ${ }^{25}$. Alors qu'Ernestine Duhamain avait au moins fini par prendre «obscurément conscience» d'un Schopenhauer à sa portée, Des Esseintes doit abandonner l'Allemand devenu impuissant. Pourquoi cet échec philosophique?

À mon sens la réponse est double, puisant à la fois dans la politique et dans la biologie. D’un côté, Des Esseintes méprise par-dessus toute autre chose «le bourgeois, rassuré, [qui] trônait, jovial, de par la force de son argent et la contagion de sa sottise. Le résultat de son avènement avait été l'écrasement de toute intelligence, la négation de toute probité, la mort de tout art $[\ldots] \gg^{26}$. Colin l'a souligné, «[s]i Des Esseintes renie son siècle, c'est que l'odieuse bourgeoisie, en une nouvelle invasion de barbares, a investi le présent ${ }^{27}$. L'exécration du bourgeois précède de peu la dernière mention de Schopenhauer dans le roman et la prépare, suggérant textuellement que c'est la montée de cette classe qui a rendu l'Allemand insuffisant même aux «intelligences choisies», en créant un monde dans lequel les esthètes sont tellement malmenés que la crise dépasse toute consolation philosophique.

Pour Smeets, «les personnages principaux que Huysmans met en scène sont avant tout célibataires, vivant dans les marges d'une société qui leur est hostile» ${ }^{28}$. Mais, Huysmans l'a lui-même signalé, Des Esseintes se sépare de ses confrères Folantin, ou même André et Cyprien d'En ménage par son esthétisme aristocratique, qui rend Schopenhauer caduc alors que Folantin avait pu le citer comme mâtre à la conclusion d'À vau-l'eau: «il faut se laisser aller à vau-l'eau; Schopenhauer a raison,

(24) J.-K. Huysmans, À rebours cit., p. 348.

(25) R.-P. Colin, Schopenhauer en France cit., p. 183.

(26) J.-K. Huysmans, À rebours cit., p. 58.

(27) R.-P. Colin, Schopenhauer en France cit., p. 187.

(28) M. SmeETs, Huysmans, Maupassant et Schopenhauer cit., p. 29. 
se dit-il, "la vie de l'homme oscille comme un pendule entre la douleur et l'ennui" »". C'est-à-dire que la différence, voulue par l'auteur, entre Folantin et Des Esseintes rapproche celui-là des personnages d'Une belle journée, et produit le même résultat quant à la narrativisation de Schopenhauer: une réussite vague et ironiquement partielle de la philosophie au dénouement, mais une réussite tout de même.

En revanche, dans À rebours, déjà dans le Chapitre VII où Huysmans avait présenté le philosophe dans son long dialogue avec Thomas à Kempis, le récit avait montré les limites de sa pensée d'une autre façon. Car ce qui suit le passage cité ci-dessus ne sont pas les indices du prétendu apaisement que Des Esseintes avait revendiqué en songeant à Schopenhauer. Tout au contraire: le chapitre enchaine avec une première crise névrotique qui annule et rend caduques les méditations qui la précèdent. Ce seront les remèdes physiques et non intellectuels qui permettront à Des Esseintes de continuer son existence solitaire pendant quelque temps, et au roman de se prolonger pendant neuf chapitres supplémentaires: «Il supprima les alcools, le café, le thé, but des laitages, recourut à des affusions d'eau froide, se bourra d'assa-foetida, de valériane et de quinine; il voulut même sortir de sa maison, se promena un peu, dans la campagne, lorsque vinrent ces jours de pluie qui la font silencieuse et vide; il se força à marcher, à prendre de l'exercice; en dernier ressort, il renonça provisoirement à la lecture $[\ldots] \gg^{30}$.

Comme le dit Jens Schnitker, «Die Obsession, mit der er seinen religios anmutenden Kult des Artifiziellen verfolgt und mit der er seine Krankheit trotz der damit verbundenen Leiden vorantreibt, verwehrt ihm dauerhafte Resignation» ${ }^{31}$. [L'obsession avec laquelle il poursuit son culte presque religieux de l'artifice, et avec laquelle il aggrave sa maladie malgré les souffrances associées, le prive d'une résignation permanente]. La résignation, dans À rebours comme dans $\grave{A}$ vau-l'eau aussi bien que dans Une belle journée, est le mot final des Schopenhauer huysmansien et céardien, mais seul parmi ces trois récits, $\grave{A}$ rebours met la résignation en scène pour la miner plutôt que la promouvoir. Non seulement la résignation n'y est-elle pas permanente, elle ne perdure pas au-delà du seul Chapitre VII. Ainsi, Colin est peut-être trop catégorique à l'égard de Huysmans en écrivant que «Schopenhauer n'a été pour Huysmans que le compagnon de route d'un moment, plaisant parce qu'il abondait dans son sens» ${ }^{32}$ étant donné que celui-ci rapprochera encore la foi chrétienne et la doctrine schopenhauerienne plus de vingt ans plus tard, mais l'évanescence du philosophe dans le texte d'À rebours, plutôt que dans l'esprit de son auteur, est claire.

Les livres du philosophe et de l'Église sont impuissants, effacés sur le plan narratologique par un régime sanitaire qui favorise le corps au dépens de l'esprit, aussi bien par l'alimentation que par l'exercice; d'ailleurs ce régime supprime, à l'étape finale, quelque livre que ce soit. Il s'ensuit que le lecteur n'a pas besoin d'attendre l'épiphanie finale de Des Esseintes pour s'apercevoir que Schopenhauer ne lui apporte pas d'aide véridique. La structure du Chapitre VII sert d'ores et déjà à enfermer Schopenhauer et le catholicisme dans un débat qui se révèle oiseux. Dans À rebours, aucun système de pensée ne réussit au protagoniste accablé par sa propre supériorité, à la différence des personnages céardiens, et c'est cela qui explique la réussite relative (soulignons l'adjectif) de Schopenhauer dans Une belle journée. Thomas West lit

(29) J.-K. Huysmans, Croquis parisiens. À vau-l'eau. Un dilemme, Paris, Stock, 1905, p. 240.

(30) ID., A rebours cit., p. 182.

(31) J. SCHNITKER, 'Sa théorie du pessimisme était la grande consolation des intelligences choisies': Schopenhauers Pessimismus in Joris-Karl Huysmans "À Rebours", "George-Jahrbuch, im Auftrag der StefanGeorge-Gesellschaft», 8, 2010, pp. 75-101, p. 88.

(32) R.-P. Colin, Schopenbauer en France cit., p. 192. 
Schopenhauer chez Huysmans sous forme de leitmotif: «The name of Schopenhauer in Huysmans' work is like a symbol, like a little daemon or Buddha, that means both pessimism and resignation and serves, as symbols do, as an element of structure, a leitmotif linking events to events, emotions to emotions $\rangle^{33}$. Pourtant, bien que le coté symbolique de Schopenhauer dans À rebours soit puissant, la notion de lier les évènements ou même les émotions s'accorde mal avec la structure narrative discutée ci-dessus, qui bien au contraire coupe les liens entre d'une part le philosophe et son symbolisme, et de l'autre le reste du roman.

Lire pour Schopenhauer dans les romans de Céard et Huysmans révèle donc de profondes affinités entre les deux auteurs quant à leur perspective philosophique. Leurs façons de comprendre les idées de l'Allemand se ressemblent remarquablement, ce qui contribue à éclairer la profondeur et la longévité de leur amitié littéraire. Mais, de par les choix de leurs cadres et personnages et, surtout, par les trames narratives employées dans Une belle journée et À rebours, l'affinité conceptuelle produit des résultats divergents dans le domaine romanesque. Cela est-il une leçon d'ordre général sur la relation entre roman et philosophie, ou est-il une question qui ne concerne que ces deux auteurs? La réponse dépasse de loin le cadre de cette étude, mais au moins pour le roman de tradition réaliste et naturaliste (et malgré la rupture avec cette tradition qu'on s'accorde typiquement à voir en À rebours), le degré d'individuation nécessaire à la composition du roman semble créer un écart entre celui-ci et les préceptes philosophiques qui viennent parfois le nourrir. Céard et Huysmans se sont donc servi de Schopenhauer pour nous donner deux portraits littéraires de la résignation, mais ces portraits ne se ressemblent qu'à moitié.

RODERICK COOKE Villanova University

(33) T. West, Schopenhauer, Huysmans and French Naturalism cit., p. 319. 